\title{
Lesões do Aquiles - Parte 2: Rupturas
}

\section{Achilles Tendon Lesions - Part 2: Ruptures}

\author{
Nacime Salomão Barbachan Mansur ${ }^{10}$ Lucas Furtado Fonseca1[ ${ }^{10}$ Fábio Teruo Matsunaga1[ \\ Daniel Soares Baumfeld ${ }^{2}{ }^{-0}$ Caio Augusto de Souza Nery ${ }^{10}$ Marcel Jun Sugawara Tamaoki ${ }^{10}$
}

${ }^{1}$ Departamento de Ortopedia e Traumatologia, Escola Paulista de
Medicina, Universidade Federal de São Paulo, Saõ Paulo, SP, Brasil
${ }^{2}$ Departamento de Ortopedia e Traumatologia, Universidade Federal
de Minas Gerais, Belo Horizonte, MG, Brasil

Rev Bras Ortop 2020;55(6):665-672.

\begin{abstract}
Endereço para correspondência Nacime Salomão Barbachan Mansur, $\mathrm{PhD}$, Departamento de Ortopedia e Traumatologia, Escola Paulista de Medicina, Universidade Federal de São Paulo, Rua Napoleão de Barros, $715,1^{\circ}$ andar, Vila Clementino, São Paulo, SP, Brasil (e-mail: nacime@uol.com.br).
\end{abstract}

\section{Resumo}

Palavras-chave

- tendão do calcâneo

- ruptura tendínea

- doença aguda

- doença crônica
A crescente incidência de rupturas do tendão calcâneo tem impactado substancialmente o cuidado ortopédico e os custos relacionados ao seu tratamento e prevenção. Motivada principalmente pelo aumento da expectativa de vida, o crescimento do uso de drogas tenotóxicas e o acesso errático à atividade física, essa lesão gera morbidade considerável aos pacientes qualquer seja o desfecho a ser considerado. A evolução das técnicas cirúrgicas e de reabilitação permitiu que ortopedistas, nos últimos anos, tivessem melhores condições para decidir a conduta mais apropriada nas roturas agudas do tendão calcâneo. Por mais que ainda frequentes pela sua alta taxa de negligência, as rupturas crônicas do Aquiles hoje encontram opções operatórias biológicas mais simples e são amparadas por um novo paradigma que se debruça sobre a especialidade.

The increasing incidence of calcaneal tendon ruptures has substantially impacted orthopedic care and costs related to its treatment and prevention. Primarily motivated by the increased life expectancy, increased use of tenotoxic drugs and erratic access to physical activity, this injury accounts for considerable morbidity regardless of its outcome. In recent years, the evolution of surgical and rehabilitation techniques gave orthopedists better conditions to decide the most appropriate conduct in acute tendon rupture. Although still frequent due to their high neglect rate, Achilles chronic ruptures currently find simpler and more biological surgical options, being supported by a new specialty-focused paradigm.

\section{Introdução}

\section{Rupturas do Tendão Calcâneo}

A peculiar anatomia do tendão calcâneo, explanada criteriosamente no capítulo 1 desses artigos de revisão (Lesões do Aquiles - Parte 1: Tendinopatias), foi essencial para o desenvolvimento do ser humano, sendo um dos grandes protagonistas no progresso do animal quadrúpede para o bípede. Com o objetivo de obter uma plataforma firme e estável para a marcha bipodal, o retropé foi rodado inferiormente para se

recebido

10 de Setembro de 2019 aceito

05 de Dezembro de 2019
DOI https://doi.org/

10.1055/s-0040-1702948. ISSN 0102-3616. acomodar no solo, fazendo do gastrocnêmio um dos últimos músculos a alongar e a ganhar potência no processo evolutivo. O Aquiles pode suportar até 12 vezes o peso corpóreo em uma corrida e é responsável por $93 \%$ do torque de força de flexão do tornozelo. ${ }^{1-3}$

A microanatomia do tendão calcâneo respeita a organização de outros tendões do corpo humano. O componente celular é formado por tenócitos e tenoblastos em até $95 \%$ dos seus elementos. Essas células têm diferentes tamanhos e formas, sendo dispostas em cadeias longas e paralelas. $\mathrm{O}$
Copyright $(2020$ by Sociedade Brasileira License terms de Ortopedia e Traumatologia. Published by Thieme Revinter Publicações Ltda, Rio de Janeiro, Brazil 
elemento extracelular é composto em 90\% por tecido colágeno, com predominância do tipo I (95\%), organizado em bandas paralelas mantidas por pequenas moléculas proteoglicanas. Cerca de $2 \%$ é elastina que confere até $200 \%$ de capacidade de deformação antes da falha. O envelhecimento e a incapacidade corpórea em prover cicatrização ideal a esses tecidos modificam essa configuração, promovendo o acúmulo de mucina, fibrina e colágeno dos tipos III e VII. ${ }^{4,5}$

A concentração de tecidos desprovidos da capacidade tênsil, elástica e biológica dos nativos enfraquece os tendões e os predispõem a rupturas macroscópicas. Autores afirmaram que essa quebra ocorreria por uma carga aplicada por uma contração muscular máxima em um tendão em sua fase de alongamento inicial. Esse risco seria potencializado por uma incapacidade na capacidade corporal de controlar contrações excessivas e não coordenadas, achados comuns em atletas que treinam de modo errático. ${ }^{6-8}$ Os mecanismos de rotura relatados são principalmente durante a fase de desprendimento (início da corrida ou salto) com o joelho em extensão (53\% dos casos), seguida pela pisada inadvertida em um buraco (17\%) e pela extensão abrupta de um tornozelo em flexão $(10 \%)^{9}$

\section{Rupturas Agudas do Tendão de Aquiles}

\section{Epidemiologia}

O tendão de Aquiles é o tendão mais frequentemente rompido do corpo humano, com uma incidência anual de 18 por 100.000 pessoas. ${ }^{10}$ Em 1575, Ambrose-Pare foi o primeiro a descrever um tratamento para ruptura aguda do Aquiles, através de enfaixamento e bandagens. ${ }^{11}$ Desde então, o tratamento não operatório era a escolha até o início do século XX, quando a cirurgia passou a ser rotineiramente indicada para as rupturas agudas do tendão calcâneo. Na década de 1920, Abrahamsen, Quenu e Stoianovitch reportaram os primeiros resultados positivos com a tenorrafia para este tipo de lesão. ${ }^{12}$

\section{Clínica}

Durante a avaliação inicial, história e exame físico completo devem ser realizados. A ruptura do tendão de Aquiles apresenta três achados clássicos ao exame clínico. Estes incluem fraqueza na flexão plantar do tornozelo, gap palpável a $\sim 4$ a $6 \mathrm{~cm}$ proximais ao calcâneo e sinal de Thompson positivo para lesão. ${ }^{13}$ As condições de partes moles devem ser avaliadas para verificar edema, hematomas, incisões prévias e integridade dos outros músculos flexores. Os pulsos são palpados e, se ausentes, a avaliação vascular deve ser considerada. Comorbidades médicas também devem ser identificadas, com ênfase em diabetes mellitus, histórico de má cicatrização de feridas e eventos tromboembólicos.

Quando a cirurgia é indicada, ela pode ser realizada em até uma ou mesmo duas semanas após a lesão, para também permitir que o aumento de volume se resolva e facilitar o posicionamento dos nós de sutura. Neste ínterim, os pacientes podem ser imobilizados em ligeiro eqüino, com ausência ou não de carga no membro, e a elevação do membro deve ser encorajada. Quando o apoio é liberado, uma bota ortopédica longa com presença de saltos em retropé deve ser utilizada.
As contraindicações para o reparo aberto incluem o status não deambulatório, doença arterial periférica grave comprometendo os tecidos moles, comorbidades médicas mal controladas e incapacidade de compreender a detalhada reabilitação pós-operatória. Tabagismo e diabetes também são contraindicações relativas devido ao aumento significativo de complicações pós-operatórias. ${ }^{14}$

\section{Propedêutica Armada}

As radiografias do tornozelo devem ser rotineiramente obtidas para se procurar fraturas do tornozelo ou da tuberosidade posterior do calcâneo, o que impõe mudanças no planejamento. $O$ borramento da gordura de Kager é um sinal indireto de lesão do tendão de Aquiles. ${ }^{15}$ A ultrassonografia (US) é o primeiro exame a ser requisitado quando há necessidade de confirmação através de imagens. $\mathrm{O}$ ultrassom pode inclusive auxiliar na decisão terapêutica. Estudo recente revelou que gaps $>10 \mathrm{~mm}$ ao primeiro exame aumentaram o risco de reruptura dentre os pacientes tratados de modo não operatório. Aqueles pacientes que seguiram o tratamento não cirúrgico e apresentaram gaps $>5 \mathrm{~mm}$ demonstraram resultados funcionais piores ao final de 12 meses. $^{16}$

Em ocasiões específicas, uma ressonância magnética (RM) poderá ser realizada para melhor se avaliar o tipo de ruptura, uma vez que lesões oblíquas e longitudinais impõem maior cuidado no planejamento das vias de acesso. Lesões associadas, como tendinopatia crônica do Aquiles são também indicações para se realizar exames mais detalhados, dado que degenerações prévias e graves podem alterar o planejamento intra e pós-operatório, como a necessidade de reforço. Por fim, a RM pode ser útil quando se identifica alterações adicionais ao exame clínico. É descrita, como exemplo, a luxação aguda do tendão tibial posterior em conjunto com a ruptura do tendão de Aquiles. ${ }^{17}$

\section{Tratamento Não Cirúrgico}

Historicamente, o tratamento não operatório tem se pautado pelas maiores taxas de re-ruptura e pela menor força flexora final. No entanto, protocolos recentes utilizando da reabilitação funcional produziram melhores resultados cinéticos e menores taxas de re-rupturas. ${ }^{18}$ Ensaios clínicos randomizados e controlados avaliaram diferentes formas de reabilitação no tratamento não cirúrgico. A carga imediata protegida com treino funcional precoce é ser recomendada (-Figura 1) aos pacientes tratados de modo conservador. ${ }^{19}$ Ela deve ser realizada com uma bota imobilizadora estável e com calços apropriados para manter o tornozelo em equino pelas 6 semanas subsequentes. Em serviços que não dispõem de uma reabilitação funcional de qualidade, o tratamento não cirúrgico deve ser visto como exceção, visto que nestas situações a cirurgia diminui o risco de re-ruptura e de perda de força. ${ }^{20}$

Outros ensaios clínicos randomizados, com pacientes alocados entre tratamento não operatório e cirurgia, com o mesmo tipo de reabilitação funcional, demonstraram resultados clínicos e funcionais similares para ambos. ${ }^{21}$ No entanto, o pico de força na avaliação isocinética demonstrou que a cirurgia (10-18\% de diferença para o lado contralateral) 


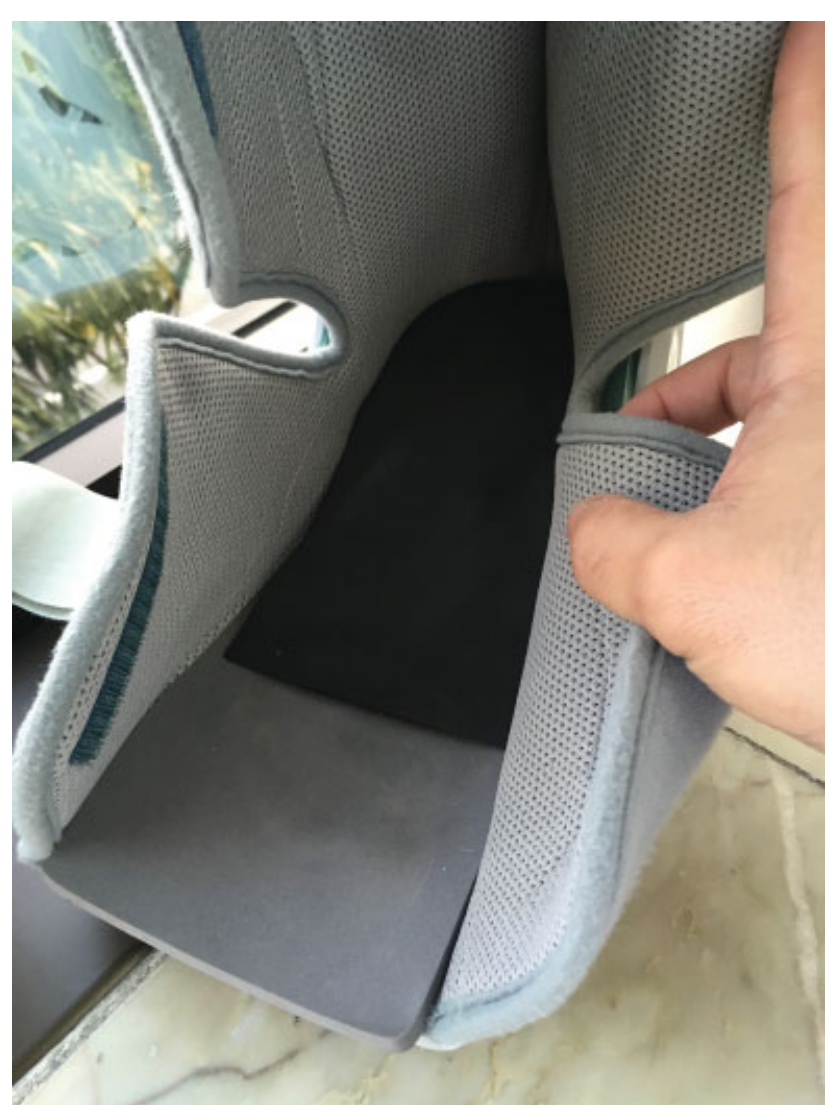

Fig. 1 Tratamento conservador funcional de uma lesão aguda do tendão calcâneo utilizando carga imediata com imobilização tipo bota e calços para manutenção do equino.

proporciona melhores índices ao longo de todos os 18 meses do período avaliado. E esta tendência é verificada em outros estudos de alta qualidade que também avaliaram a força muscular pormenorizadamente. ${ }^{22-24}$ De fato, a grande maioria dos pacientes apresentará maior perda de força de flexão plantar quando tratados não cirurgicamente. No entanto, esta diferença não compromete suas atividades de vida diária, especialmente em pacientes não atletas e tratados de forma funcional. ${ }^{22-24}$

\section{Tratamento Cirúrgico}

O tratamento das rupturas agudas do tendão de Aquiles é controverso e sem consenso na literatura no que diz respeito à abordagem (não operatória versus a cirúrgica) e a técnica ideal para a realização do tratamento operatório. ${ }^{25}$ Uma revisão da Cochrane avaliando as diferenças entre estas condutas relatou que o reparo cirúrgico significativamente reduz o risco de re-ruptura do tendão de Aquiles, não obstante as maiores taxas de complicações, incluindo infecção da ferida operatória. ${ }^{26}$ Diretrizes apresentadas pela Academia Americana de Ortopedia (AAOS, na sigla em inglês) determinam moderada evidência de que a abordagem não operatória apresenta menos complicações, com maiores taxas de re-rupturas em relação ao reparo cirúrgico, e que as técnicas que utilizam incisões menores, ou minimamente invasivas, apresentam menores taxas de complicações quando comparadas ao reparo aberto. ${ }^{27}$
0 reparo aberto tradicional consiste em 5 uma incisão posteromedial longitudinal de 5 a $8 \mathrm{~cm}$ sobre o foco da ruptura, com dissecção do paratendão, evacuação do hematoma, desbridamento dos cotos tendíneos, e colocação de suturas tipo Krackow para atamento dos fios "boca a boca" e cooptação dos cotos. Embora esta técnica de reparo seja biomecanicamente forte e apresente bons resultados em geral, tem sido associada à deiscência de ferida superficial e profundamente. Neste contexto, as técnicas "mini-open" são atrativas, uma vez que minimizam o dano às partes moles, alcançam um reparo sólido e firme do tipo direto. Essas características permitiriam ganho funcional e menores

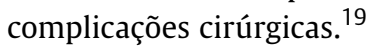

O sistema percutâneo de reparação de Aquiles (PARS, na sigla em inglês) (Arthrex Inc., Naples, FL, EUA) é uma técnica moderna e mini-aberta que utiliza uma incisão cutânea transversal de $\sim 2 \mathrm{~cm}$ em combinação com a introdução de um aparato de metal ligeiramente curvo por dentro do paratendão para passar as suturas de bloqueio. A técnica permite abertura mínima dos planos profundos e confere grande estabilidade ao tendão suturado (-Figura 2). Recentemente, a técnica PARS demonstrou acelerar a recuperação e o tempo de retorno, assim como apresentar menores taxas de deiscência de ferida operatória. ${ }^{28}$

Uma comparação biomecânica do reparo tipo PARS com o "mini-open" utilizando apenas suturas não bloqueadas

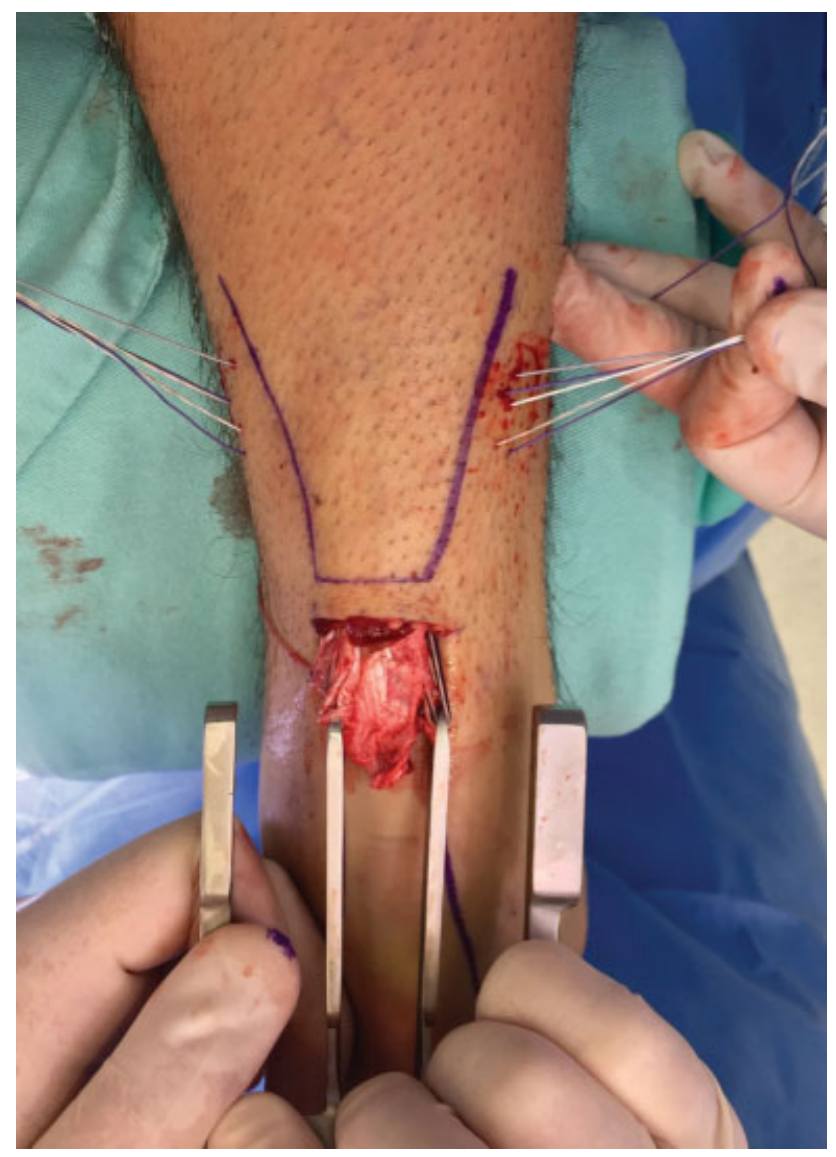

Fig. 2 Tenorrafia de uma ruptura aguda do Aquiles com um método minimamente invasivo. Os garfos são introduzidos nos cotos proximais dentro do paratendão e as suturas passadas pelo dispositivo. 
constatou que o PARS teve maior força em termos de carregamento cíclico e carga até a falha. ${ }^{29}$ Uma grande série recente comparando 101 PARS e 169 reparos tradicionais abertos de Aquiles relatou que o reparo tipo PARS apresentou tempos operatórios significativamente mais curtos e um número maior de pacientes capazes de retornar às atividades físicas regulares aos 5 meses após a cirurgia, em comparação com o reparo aberto. A taxa global de complicações pósoperatórias no PARS foi de $5 \%$, enquanto o reparo aberto apresentou $11 \%$, em sua maioria devido às deiscências e infecções. Não houve casos de re-ruptura, neurite do sural, ou infecção profunda no grupo PARS. ${ }^{26}$

Em relação ao reforço agudo nas rupturas do tendão calcâneo, os estudos atuais não suportam o seu uso. Uma revisão sistemática e meta-análise recente demonstrou em 169 participantes ( 83 com reforço agudo e 86 com tenorrafia isolada do Aquiles) que não houve diferenças quanto à satisfação, taxas de re-rupturas e complicações. ${ }^{28}$

Importante ser notado que a literatura também vem favorecendo a reabilitação precoce e funcional, em detrimento da imobilização isolada nas primeiras semanas nos pacientes operados, independentemente da técnica utilizada. ${ }^{29} \mathrm{Em}$ linhas gerais, o protocolo pós-operatório para ambas as abordagens cirúrgicas, "mini-open" e aberta, é o mesmo. Os pacientes são mantidos em flexão plantar de $\sim 20^{\circ}$, ou simétrica ao lado sadio, por 2 semanas. A seguir, a bota removível é colocada com os calços específicos para Aquiles. ${ }^{30}$ Os pontos são deixados por 3 semanas, e o equino reduzido da $4^{\underline{a}}$ até a $6^{\underline{a}}$ semana. Acreditamos que seja crucial a proteção do Aquiles durante as 4 primeiras semanas, em razão do potencial de alongamento do tendão. Em pacientes atletas, início de carga em equino na $2^{\underline{a}}$ semana demonstrou ser uma abordagem segura e de rápido retorno ao esporte. ${ }^{17} \mathrm{O}$ objetivo é que os pacientes estejam com carga total plantígrada ao final da $6^{a}$ ou $7^{\mathfrak{a}}$ semana. Entre as semanas 8 e 12 , os pacientes passam a utilizar sapatos regulares, evitando-se a extensão do tornozelo além do neutro, com o uso de salto interno no calçado, para elevação do retropé. Atividades como corrida e salto são normalmente permitidas após 16 semanas, e o retorno total ao esporte ocorre entre os meses 5 e 6 .

Caberá ao cirurgião decidir qual técnica cirúrgica a ser utilizada, baseado em sua análise individual de cada paciente, e especialmente em sua capacidade técnica e experiência. Todavia, deve-se levar em consideração as evidências crescentes de que as técnicas menos invasivas podem ser superiores ao tratamento clássico para as rupturas agudas do tendão de Aquiles. $\mathrm{O}$ resumo das indicações das modalidades de tratamento baseado nas evidências científicas atuais encontra-se na - Tabela 1.

Tabela 1 Grau de recomendação dos tratamentos para a ruptura aguda do Aquiles

\begin{tabular}{|l|l|}
\hline Modalidade & Grau de Recomendação \\
\hline Tratamento Conservador & B \\
\hline Reparo Aberto & A \\
\hline Reparo “Mini-Open” & A \\
\hline
\end{tabular}

\section{Rupturas Crônicas do Tendão de Aquiles}

\section{Epidemiologia}

Apesar de comum, a ruptura do Aquiles ainda apresenta uma taxa de falha no diagnóstico à primeira avaliação médica de 20 a $25 \%$, ocasionando um atraso no seu diagnóstico e tratamento, concorrendo para um aumento na prevalência de casos crônicos. ${ }^{31,32}$ Essa alta porcentagem é causada tanto pela falta de procura por atendimento desses pacientes (muitos creditam uma lesão muscular ou de menor gravidade), pela incapacidade de acesso de muitos indivíduos a serviços especializados de saúde e pela deficiência de profissionais em realizar o correto diagnóstico precoce. ${ }^{33,34}$

Alguns critérios podem ser utilizados para a definição da lesão crônica como a existência de tecido fibrocicatricial interposto entre seus cotos, a presença de largo defeito com afastamento e retração dos mesmos, um período decorrido da lesão até o momento do diagnóstico $>6$ semanas ou sintomas de fraqueza e dificuldade para caminhar e subir escadas. $^{31,32,35}$

\section{Fisiopatogenia}

A rotura do tendão provoca uma separação natural entre as extremidades. A partir do momento da lesão, em decorrência do sangramento local e da sinalização inflamatória regional, tecido reparativo começa a ser formado. ${ }^{36} \mathrm{~A}$ persistência de contração muscular e mobilização local promove uma migração proximal do tríceps e a conseguinte aderência dos cotos no paratendão e tecidos adjacentes. ${ }^{31}$ $\mathrm{Na}$ zona da ruptura forma-se comumente um tecido fibrocicatricial alongado e que carece de competência mecânica. Em alguns casos, nenhuma cicatrização se forma e um grande defeito se estabelece. Qualquer que seja o desfecho, o tríceps sural perde substancialmente a força pelo alongamento da unidade musculotendínea ou pela ausência de comunicação entre origem e inserção. ${ }^{37,38}$

\section{Clínica}

Em efeito do tempo decorrido após a lesão inicial, muitos pacientes não reclamam de dor ou edema na região posterior da perna. A evolução do quadro leva comumente esses indivíduos a procurarem atendimento pela fraqueza na flexão plantar do tornozelo. Ainda que presente pela integralidade dos flexores secundários, essa força se encontra extremamente amortizada pelo protagonismo funcional do tríceps sural. Esse prejuízo afeta de modo direto as atividades do cotidiano dos seus portadores, pois chega a incapacitá-los a ficar na ponta dos pés ou de caminhar com qualidade. ${ }^{31,39}$

A rica propedêutica de uma ruptura aguda pode não estar presente nas lesões crônicas. É possível observar uma marcha irregular, com encurtamento da passada ipsilateral, aumento da fase intermediária e do segundo rolamento, além de ausência de desprendimento de qualidade no membro. 0 clássico gap pode não ser palpável pela presença de tecido cicatricial na região, o que também pode confundir o teste de Thompson. A hipotrofia da musculatura da perna é evidente e o teste de Matles mostra assimetria entre os lados avaliados, corroborando a presença da lesão. ${ }^{38,39}$ 


\section{Propedêutica Armada}

Apesar de o diagnóstico ser essencialmente clínico, alguns exames subsidiários auxiliam no desenho do quadro do paciente e no planejamento do tratamento. Radiografias simples em perfil podem traduzir rupturas insercionais geradoras de avulsões da tuberosidade posterior do calcâneo. A RM do tornozelo é de uso restrito apenas aos casos de dúvida diagnóstica. A US tem sido utilizada como método de avaliação do alongamento musculotendíneo, ainda com resultados incipientes. 0 estudo isocinético pode contribuir na determinação da fraqueza clínica de pacientes com quadros mais frustros e auxiliar o profissional na decisão terapêutica. $^{39,40}$

A evolução do conhecimento torna a realização de uma RM da perna (bilateral comparativa) uma importante arma em pacientes com rupturas crônicas do Aquiles. Estudos prévios demonstraram alterações no ângulo de bipenação e substituição gordurosa da musculatura com o decorrer da condição, como discutido no capítulo de tendinopatias (Lesões do Aquiles - Parte 1: Tendinopatias). Lipossubstituições grau 0 e 1 (classificação de Goutalier adaptada) no tríceps sural possibilitam o investimento em procedimentos reconstrutivos no tendão, pois o músculo ainda é funcional. Degenerações gordurosas grau 2, 3 ou 4 determinam procedimentos de substituição, com o uso de transferências tendíneas, visto que o complexo muscular gastrosóleo apresenta perda irreversível nessas ocasiões. ${ }^{39,41}$

\section{Tratamento Não Cirúrgico}

Praticamente não há espaço para o tratamento conservador em pacientes com rupturas crônicas do Aquiles pelo alto grau de limitação imprimido com a fraqueza do compartimento superficial posterior da perna. A condução não operatória deve ser reservada a pacientes com comorbidades graves e contraindicações absolutas a qualquer cirurgia. O tratamento conservador é baseado no fortalecimento dos flexores secundários do tornozelo (hálux, dedos, fibulares, tibial posterior) e na utilização de órteses dorsais e anteriores (AFO) que limitam a hiperextensão do tornozelo e a marcha calcânea. ${ }^{31,33}$

Mesmo os pacientes com baixa demanda ou com doenças de base que prejudicam a cicatrização tecidual (vasculopatias, diabetes, tabagistas, doenças reumáticas...) podem ser beneficiados de modo substancial por um procedimento, ademais quando se considera as opções menos invasivas disponíveis na atualidade. A necessidade cirúrgica em pacientes com este perfil, devido a incapacidade funcional decorrendo do quadro, é mais um forte argumento a favor de políticas de prevenção de perdas diagnósticas. Essa população poderia usufruir de um resultado efetivo se o tratamento não cirúrgico correto fosse a eles oferecido na lesão inicial. ${ }^{41,42}$

\section{Tratamento Cirúrgico}

Tradicionalmente, a decisão sobre a técnica cirúrgica tem sido baseada no tamanho do defeito observado durante a cirurgia após a limpeza e liberação dos cotos. Esses estadiamentos são baseados em opiniões de especialistas (nível V) e as cirurgias propostas encontram amparo apenas em estudos nível IV de evidência. Ainda não há consenso sobre a melhor técnica para essas rupturas. ${ }^{37,43}$

Falhas pequenas (até $2 \mathrm{~cm}$ ) poderiam ser tratadas com suturas boca-a-boca após liberações do compartimento posterior. Defeitos moderados ( 2 a $6 \mathrm{~cm}$ ) seriam manejados com alongamento $\mathrm{V}-\mathrm{Y}$, retalhos locais ou transferências tendíneas. Lesões maiores ( $>6 \mathrm{~cm}$ ) requereriam o uso de aloenxertos, enxertos sintéticos, liberações, retalhos ou a combinação desses métodos. ${ }^{43,44}$ Entretanto, essas abordagens clássicas possuem taxas de complicações que se aproximam dos 72\%, compreendendo deiscências, infecções, morbidade doadora e perda de força. 0 alto índice de problemas em decorrência das grandes vias de acesso e das aderências nessas cirurgias tem levado pesquisadores a procurar opções de menor agressividade local. ${ }^{45}$

As produções atuais caminham para procedimentos de que respeitam a biologia local e a qualidade da musculatura do complexo gastrosóleo. Na presença de lipossubstituições grau 0 ou 1, tentativas de salvamento da unidade muscular através da reconstrução do tendão são válidas. Nas degenerações grau 2, 3 ou 4, o quadro irrevogável do músculo obriga o cirurgião a buscar substitutos para o tríceps sural. ${ }^{46-48}$

Maffulli et al. ${ }^{45}$ publicaram artigos utilizando enxertos livres na reconstrução de tendões de Aquiles cronicamente rompidos com uso de pequenas incisões e preservação da ponte de pele sobre a falha. Relataram bons resultados e abriram possibilidades para técnicas menos mórbidas na região. O coto proximal do tendão (em um músculo com qualidade) é preparado com o enxerto livre (semitendíneo, por exemplo) e essa reconstrução é fixada na região posterior e distal da tuberosidade posterior do calcâneo (-Figura 3) através de um túnel com um parafuso de interferência. ${ }^{45,49}$

Diferentes tendões doadores são relatados para transferências nas rupturas crônicas do tendão calcâneo. Hoje, entende-se que essa indicação é mais voltada para músculos que apresentam degeneração gordurosa e não possuem funcionalidade para uma possível reconstrução. A escolha entre flexor longo do hálux (FLH), flexor longo dos dedos ou fibular curto deve ser baseada na qualidade dos tecidos locais, o perfil de cada paciente e as perdas previstas com a transposição (perda de toe-off e força de arranque, perda de força dos dedos menores, perda de um estabilizador lateral secundário do tornozelo). Nenhuma das técnicas mostrou-se superior a outra, em que pese a maior constância do FLH nas publicações e na preferência de muitos autores. Técnicas de menor invasividade também têm sido utilizadas para as transferências, com destaque para a possibilidade de transposição endoscópica (-Figura 4) do $\mathrm{FLH}{ }^{50-54} \mathrm{O}$ resumo das indicações das modalidades de tratamento baseado nas evidências científicas atuais encontra-se na -Tabela 2.

\section{Considerações Finais}

As últimas duas décadas foram determinantes para o progresso no conhecimento sobre as rupturas agudas do tendão de Aquiles. A evolução das técnicas cirúrgicas, do tratamento não operatório e da reabilitação trouxe luz a um tema de 


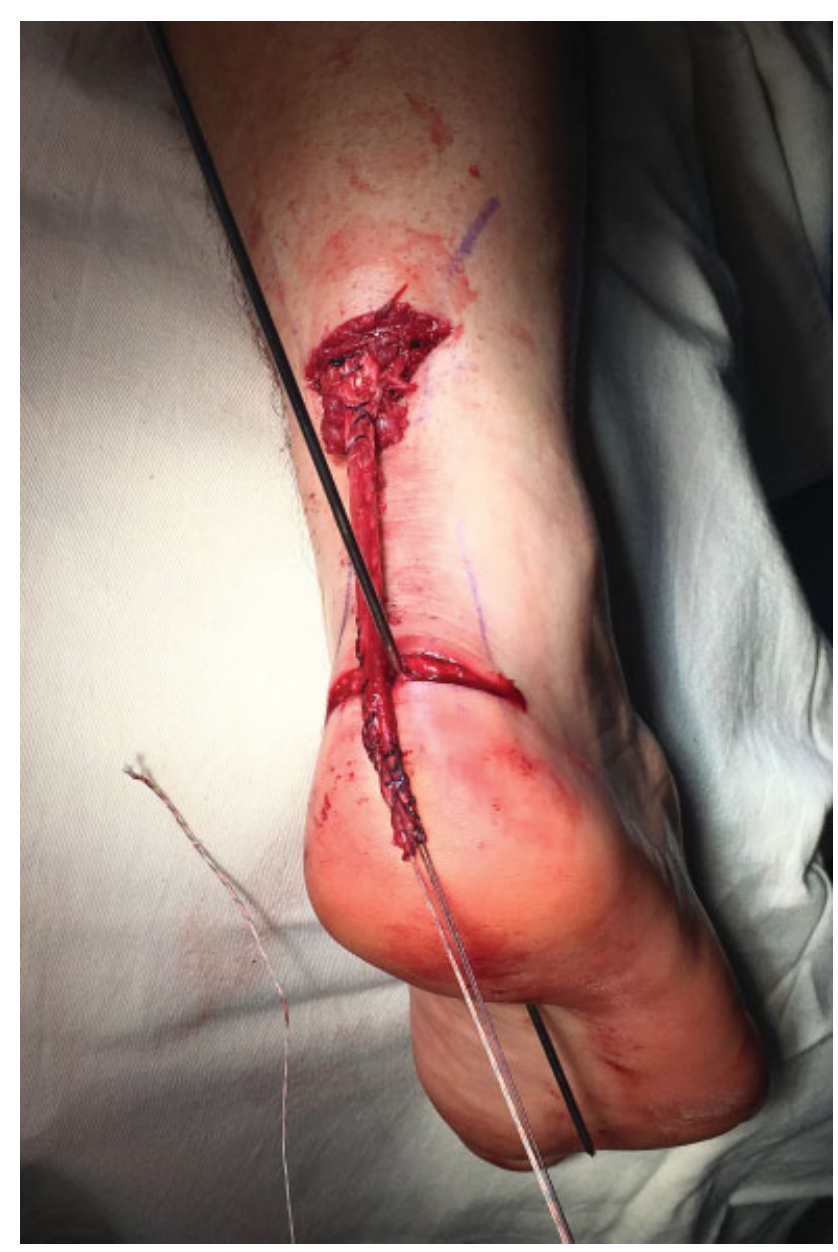

Fig. 3 Reconstrução de uma lesão crônica do tendão calcâneo com autoenxerto livre de semitendíneo em um músculo com viabilidade.

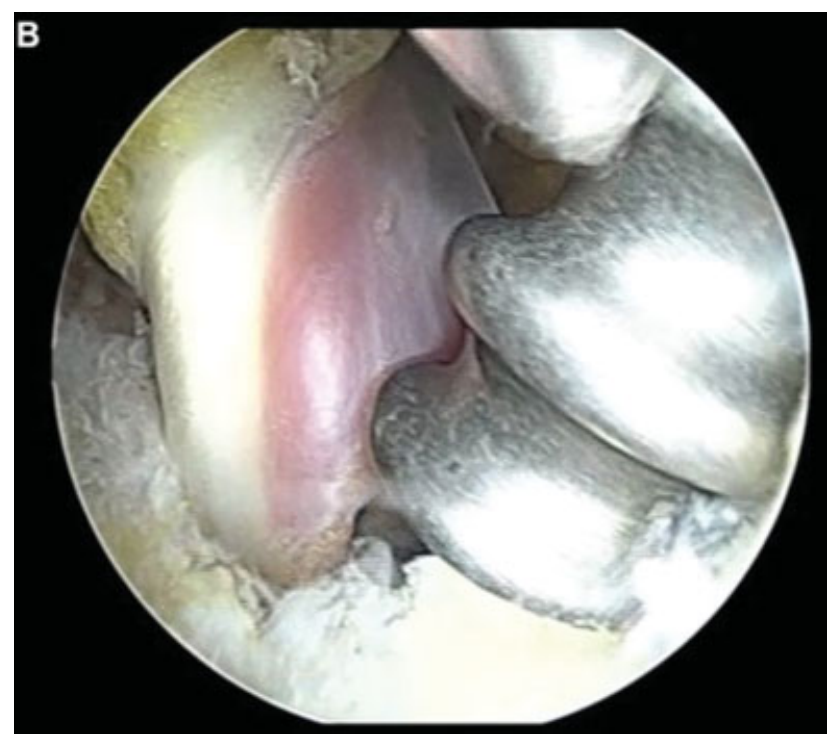

Fig. 4 Transferência endoscópica do flexor longo do hálux para a tuberosidade posterior do calcâneo em um paciente com lesão crônica do Aquiles e tríceps apresentado com lipossubstituição muscular.

interesse e incidência crescente. Os excelentes resultados que podem ser obtidos com as modalidades terapêuticas atuais proporcionam ao profissional boas condições na decisão da melhor conduta para um específico paciente. As
Tabela 2 Grau de recomendação dos tratamentos para a ruptura crônica do Aquiles

\begin{tabular}{|l|l|}
\hline Modalidade & $\begin{array}{l}\text { Grau de } \\
\text { Recomendação }\end{array}$ \\
\hline Transferência Aberta do FLH ou FC & C \\
\hline Reconstrução em VY & C \\
\hline Reconstrução em “Turn Down Flaps” & C \\
\hline Transferência Endoscópica do FLH & I \\
\hline Reconstrução com Enxerto Livre (ST/G) & C \\
\hline
\end{tabular}

Abreviações: FC: Fibular Curto; FLH: Flexor Longo do Hálux; G: Grácil; ST: Semitendíneo.

publicações correntes ofertam grau de recomendação B para o tratamento não cirúrgico funcional e grau $\mathrm{A}$ para tanto a tenorrafia aberta quanto a minimamente invasiva. Qualquer que seja a escolha, ela deve ser seguida por um protocolo de reabilitação dinâmico, que contemple carga e mobilidade controlada precoce.

Ainda que menos exuberante, enriquecimento também tem sido observado na ciência das rupturas crônicas do Aquiles. Políticas de prevenção de perdas diagnósticas, o que diminuiria a demanda de grandes procedimentos reconstrutivos, permanecem praticamente inexistentes. Entretanto, a morbidade das técnicas tradicionais tem sido suplantada por cirurgias com um respeito maior à biologia local e à qualidade do músculo disponível. Mesmo observando um grau máximo de recomendação $C$ para esses tratamentos operatórios, esses procedimentos têm permitido que essa população alcance resultados funcionais mais animadores e muito superiores aos relatados no passado.

\section{Conflito de Interesses}

Os autores declaram não haver conflito de interesses.

\section{Referências}

1 Amis J. The gastrocnemius: a new paradigm for the human foot and ankle. Foot Ankle Clin 2014;19(04):637-647

2 Komi PV. Relevance of in vivo force measurements to human biomechanics. J Biomech 1990;23(Suppl 1):23-34

3 Schmitt D. Insights into the evolution of human bipedalism from experimental studies of humans and other primates. J Exp Biol 2003;206(Pt 9):1437-1448

4 Doral MN, Alam M, Bozkurt M, et al. Functional anatomy of the Achilles tendon. Knee Surg Sports Traumatol Arthrosc 2010;18 (05):638-643

5 Maffulli N, Barrass V, Ewen SWB. Light microscopic histology of achilles tendon ruptures. A comparison with unruptured tendons. Am J Sports Med 2000;28(06):857-863

6 Barfred T. Achilles tendon rupture. Aetiology and pathogenesis of subcutaneous rupture assessed on the basis of the literature and rupture experiments on rats. Acta Orthop Scand Suppl 1973; Suppl 152:3-126

7 Inglis AE, Sculco TP. Surgical repair of ruptures of the tendo Achillis. Clin Orthop Relat Res 1981;(156):160-169

8 Egger AC, Berkowitz MJ. Achilles tendon injuries. Curr Rev Musculoskelet Med 2017;10(01):72-80

9 Gross CE, Nunley JA 2nd. Acute Achilles Tendon Ruptures. Foot Ankle Int 2016;37(02):233-239 
10 Miller CP, Chiodo CP. Open Repair of Achilles Tendon Ruptures. Tech Foot Ankle Surg 2017;16:62-67

11 Cetti R, Christensen SE, Ejsted R, Jensen NM, Jorgensen U. Operative versus nonoperative treatment of Achilles tendon rupture. A prospective randomized study and review of the literature. Am J Sports Med 1993;21(06):791-799

12 Abrahamsen K. Ruptura Tendinis Achillis. Ugeskr Laeger 1923; 85:279-285

13 Hattrup SJ, Johnson KA. A review of ruptures of the Achilles tendon. Foot Ankle 1985;6(01):34-38

14 Lantto I, Heikkinen J, Flinkkila T, et al. A Prospective Randomized Trial Comparing Surgical and Nonsurgical Treatments of Acute Achilles Tendon Ruptures. Am J Sports Med 2016;44(09):2406-2414

15 Demetracopoulos CA, Gilbert SL, Young E, Baxter JR, Deland JT. Limited-Open Achilles Tendon Repair Using Locking Sutures Versus Nonlocking Sutures: An In Vitro Model. Foot Ankle Int 2014;35(06):612-618

16 Westin O, Nilsson Helander K, Grävare Silbernagel K, Möller M, Kälebo P, Karlsson J. Acute Ultrasonography Investigation to Predict Reruptures and Outcomes in Patients With an Achilles Tendon Rupture. Orthop J Sports Med 2016;4(10):2325967116667920

17 McCullough KA, Shaw CM, Anderson RB. Mini-open repair of achilles rupture in the national football league. J Surg Orthop Adv 2014;23(04):179-183

18 Wilkins R, Bisson LJ. Operative versus nonoperative management of acute Achilles tendon ruptures: a quantitative systematic review of randomized controlled trials. Am J Sports Med 2012; 40(09):2154-2160

19 Barfod KW, Bencke J, Lauridsen HB, Ban I, Ebskov L, Troelsen A. Nonoperative dynamic treatment of acute achilles tendon rupture: the influence of early weight-bearing on clinical outcome: a blinded, randomized controlled trial. J Bone Joint Surg Am 2014; 96(18):1497-1503

20 Soroceanu A, Sidhwa F, Aarabi S, Kaufman A, Glazebrook M. Surgical versus nonsurgical treatment of acute Achilles tendon rupture: a meta-analysis of randomized trials. J Bone Joint Surg Am 2012;94(23):2136-2143

21 Deng S, Sun Z, Zhang C, Chen G, Li J. Surgical Treatment Versus Conservative Management for Acute Achilles Tendon Rupture: A Systematic Review and Meta-Analysis of Randomized Controlled Trials. J Foot Ankle Surg 2017;56(06):1236-1243

22 Willits K, Amendola A, Bryant D, et al. Operative versus nonoperative treatment of acute Achilles tendon ruptures: a multicenter randomized trial using accelerated functional rehabilitation. J Bone Joint Surg Am 2010;92(17):2767-2775

23 Keating JF, Will EM. Operative versus non-operative treatment of acute rupture of tendo Achillis: a prospective randomised evaluation of functional outcome. J Bone Joint Surg Br 2011;93(08): 1071-1078

24 Nilsson-Helander K, Silbernagel KG, Thomeé R, et al. Acute achilles tendon rupture: a randomized, controlled study comparing surgical and nonsurgical treatments using validated outcome measures. Am J Sports Med 2010;38(11):2186-2193

25 Khan RJ, Fick D, Keogh A, Crawford J, Brammar T, Parker M. Treatment of acute achilles tendon ruptures. A meta-analysis of randomized, controlled trials. J Bone Joint Surg Am 2005;87(10): 2202-2210

26 Hsu AR, Jones CP, Cohen BE, Davis WH, Ellington JK, Anderson RB. Clinical Outcomes and Complications of Percutaneous Achilles Repair System Versus Open Technique for Acute Achilles Tendon Ruptures. Foot Ankle Int 2015;36(11):1279-1286

27 Chiodo CP, Glazebrook M, Bluman EM, et al. American Academy of Orthopaedic Surgeons. American Academy of Orthopaedic Surgeons clinical practice guideline on treatment of Achilles tendon rupture. J Bone Joint Surg Am 2010;92(14):2466-2468

28 Zhang YJ, Zhang C, Wang Q, Lin XJ. Augmented Versus Nonaugmented Repair of Acute Achilles Tendon Rupture: A Systematic Review and Meta-analysis. Am J Sports Med 2018;46(07):1767-1772
29 Wagner P, Wagner E, López M, Etchevers G, Valencia O, GuzmánVenegas R. Proximal and Distal Failure Site Analysis in Percutaneous Achilles Tendon Rupture Repair. Foot Ankle Int 2019;40 (12):1424-1429

30 Ellison P, Molloy A, Mason LW. Early Protected Weightbearing for Acute Ruptures of the Achilles Tendon: Do Commonly Used Orthoses Produce the Required Equinus? J Foot Ankle Surg 2017;56(05):960-963

31 Padanilam TG. Chronic Achilles tendon ruptures. Foot Ankle Clin 2009;14(04):711-728

32 Malagelada F, Clark C, Dega R. Management of chronic Achilles tendon ruptures-A review. Foot 2016;28:54-60

33 Maffulli N, Ajis A, Longo UG, Denaro V. Chronic rupture of tendo Achillis. Foot Ankle Clin 2007;12(04):583-596, vi

34 Den Hartog BD. Surgical strategies: delayed diagnosis or neglected achilles' tendon ruptures. Foot Ankle Int 2008;29(04):456-463

35 Vernois J, Bendall S, Ferraz L, Redfern D. Arthroscopic FHL harvest and transfer for neglected TA rupture. Tech Foot Ankle Surg 2016; 15(01):32-38

36 Carden DG, Noble J, Chalmers J, Lunn P, Ellis J. Rupture of the calcaneal tendon. The early and late management. J Bone Joint Surg Br 1987;69(03):416-420

37 Myerson MS. Achilles tendon ruptures. Instr Course Lect 1999; 48:219-230

38 Schweitzer KM Jr, Dekker TJ, Adams SB. Chronic Achilles Ruptures: Reconstructive Options. J Am Acad Orthop Surg 2018;26(21): 753-763

39 Steginsky BD, Van Dyke B, Berlet GC. The Missed Achilles Tear: Now what? Foot Ankle Clin 2017;22(04):715-734

40 de Cesar Netto C, Chinanuvathana A, Fonseca LFD, Dein EJ, Tan EW, Schon LC. Outcomes of flexor digitorum longus (FDL) tendon transfer in the treatment of Achilles tendon disorders. Foot Ankle Surg 2019;25(03):303-309

41 Maffulli N, Oliva F, Maffulli GD, Buono AD, Gougoulias N. Surgical management of chronic Achilles tendon ruptures using less invasive techniques. Foot Ankle Surg 2018;24(02):164-170

42 Maffulli N, Via AG, Oliva F. Chronic Achilles Tendon Rupture. Open Orthop J 2017;11(01):660-669

43 Kuwada GT. Classification of tendo Achillis rupture with consideration of surgical repair techniques. J Foot Surg 1990;29(04): 361-365

44 Maffulli N, Loppini M, Longo UG, Maffulli GD, Denaro V. Minimally invasive reconstruction of chronic achilles tendon ruptures using the ipsilateral free semitendinosus tendon graft and interference screw fixation. Am J Sports Med 2013;41(05):1100-1107

45 Maffulli N, Longo UG, Spiezia F, Denaro V. Free hamstrings tendon transfer and interference screw fixation for less invasive reconstruction of chronic avulsions of the Achilles tendon. Knee Surg Sports Traumatol Arthrosc 2010;18(02):269-273

46 Fischer MA, Pfirrmann CW, Espinosa N, Raptis DA, Buck FM. Dixon-based MRI for assessment of muscle-fat content in phantoms, healthy volunteers and patients with achillodynia: comparison to visual assessment of calf muscle quality. Eur Radiol 2014; 24(06):1366-1375

47 Hoffmann A, Mamisch N, Buck FM, Espinosa N, Pfirrmann CW, Zanetti M. Oedema and fatty degeneration of the soleus and gastrocnemius muscles on MR images in patients with Achilles tendon abnormalities. Eur Radiol 2011;21(09): 1996-2003

48 Rahm S, Spross C, Gerber F, Farshad M, Buck FM, Espinosa N. Operative treatment of chronic irreparable Achilles tendon ruptures with large flexor hallucis longus tendon transfers. Foot Ankle Int 2013;34(08):1100-1110

49 Asaumi I, Nery C, Raduan F, Mansur N, Apostolico Netto A, Lo Turco D. A modified Maffulli technique for Achilles Tendon lesions. Foot Ankle Surg 2017;23:151

50 Baumfeld D, Baumfeld T, Figueiredo AR, et al. Endoscopic Flexor Halluces Longus transfer for Chronic Achilles Tendon rupture - 
672 Lesões do Aquiles - Parte 2: Rupturas Mansur et al.

technique description and early post-operative results. Muscles Ligaments Tendons J 2017;7(02):341-346

51 Wapner KL, Pavlock GS, Hecht PJ, Naselli F, Walther R. Repair of chronic Achilles tendon rupture with flexor hallucis longus tendon transfer. Foot Ankle 1993;14(08):443-449

52 Oksanen MM, Haapasalo HH, Elo PP, Laine HJ. Hypertrophy of the flexor hallucis longus muscle after tendon transfer in patients with chronic Achilles tendon rupture. Foot Ankle Surg 2014;20 (04):253-257

53 Den Hartog BD. Flexor hallucis longus transfer for chronic Achilles tendonosis. Foot Ankle Int 2003;24(03):233-237

54 Gossage W, Kohls-Gatzoulis J, Solan M. Endoscopic assisted repair of chronic achilles tendon rupture with flexor hallucis longus augmentation. Foot Ankle Int 2010;31(04):343-347 